\title{
The physiology and management of childhood asthma
}

\author{
R. S. JONES \\ M.D., M.R.C.P., D.C.H. \\ Consultant Clinical Physiologist, Alder Hey Children's Hospital, Liverpool, \\ and Director of Studies, Institute of Child Health, University of Liverpool
}

THE MANY clinical patterns which occur in asthma and the unpredictable course of the disease have made it notoriously difficult to treat and have led to widely differing views on management. The arrival of ventilatory function tests increased the accuracy of clinical assessment in some respects but has confirmed the impression that considerable fluctuations in ventilatory function occur over quite short periods of time, making objective appraisal difficult even with the help of these tests. Nevertheless, with their aid it is now possible to place much of the therapy on a rational basis.

\section{Patho-physiology}

Asthma is a disease of the bronchioles. Inhaled antigenic material passes across the surface-lining film and mucosal layer to react with reaginic material adherent to the mast cells lying between the mucosal and bronchiolar muscle layers causing release of histamine and other constrictor agents. The link between the antigen-antibody reaction on the one hand and on the other, bronchiolar muscle contraction together with the mucosal response which includes the secretion of large amounts of eosinophilcontaining mucoid material, is not yet fully understood (Amos, 1971).

The consequence, however, is an increase in airways resistance which causes a secondary increase in lung volume compartments, especially the residual capacity. An important key to the interpretation of clinical events is an appreciation of the fact that the increase of airways resistance (AR) is made up of two components which behave quite differently. First, there is a relatively fixed component due primarily to mucosal swelling and luminal obstruction caused by secretion. Secondly, there is a labile component due primarily to bronchiolar muscle contraction. Since many diseases may cause a fixed increase in AR, from the point of view of physiology, asthma must be defined in terms of the labile component (Jones, 1966). An abnormally labile bronchial tree is therefore the specific abnormality which distinguishes asthma from other pulmonary diseases. In offering a clinical definition of asthma, Scadding (1969) is referring to this labile component of AR.

\section{Classification}

A rational physiological classification in terms of these two components of AR is used here as a basis for the discussion of management. The FEV (Forced Expiratory Volume in $1 \mathrm{sec}$ ) is used to obtain an index of these components (Jones, 1966).

The $\mathrm{FEV}_{1}$ is measured with the subject at rest. Maximum bronchoconstriction following a period of $8 \mathrm{~min}$ exercise is measured in terms of the fall of $\mathrm{FEV}_{1}$ below the value at rest. After return to the resting level, maximum bronchodilatation is obtained by giving isoprenaline as an aerosol followed by brisk exercise of $2 \mathrm{~min}$ duration. The difference between the highest and the lowest values for $F_{1}$ are expressed as a percentage of the predicted normal. Hence,

Lability Index $=\frac{\text { Fall of } \mathrm{FEV}_{1}+\mathrm{Rise}_{\text {of }} \mathrm{FEV}_{1}}{\text { Predicted normal FEV }} \underset{1}{100}$

Using this technique childhood asthmatics can be divided into three groups (Table 1). Group 3 subjects

Table 1. Classification

\begin{tabular}{crc}
\hline Group & Max FEV $_{1}$ & Lability \\
\hline 1 & $\mathrm{~N}$ & $15-30 \%$ \\
2 & $\mathrm{~N}$ & $>30 \%$ \\
3 & $<\mathrm{N}$ & Variable \\
Normal & $\mathrm{N}$ & $<20 \%$ \\
\hline
\end{tabular}

Lability Index $=$

Fall of $\mathrm{FEV}_{1}$ in litres + Rise of $\mathrm{FEV}_{1}$ in litres

$$
\text { Predicted normal FEV }
$$

In the normal subject, the $\mathrm{FEV}_{1}$ at rest replaces the predicted normal FEV $_{1}$.

Fall of $F_{E V}=$ Decrease below resting level after $8 \mathrm{~min}$ exercise.

Rise of $\mathrm{FEV}_{1}=$ Rise above resting level after bronchodilator drug + exercise for $2 \mathrm{~min}$.

are relatively severe asthmatics with an increase in the fixed component of AR sufficient to prevent bronchodilatation to within the normal limits of $F_{E V}$. In the remainder, the fixed component is 
relatively unimportant and they are divided arbitrarily into those in whom the labile component is relatively small (Group 1), and those in whom it is large (Group 2).

It is essential to appreciate that a highly labile bronchial tree (Group 2), may or may not result in clinical asthma. Psychological or other stimuli may cause mild or severe attacks or may fail to cause an attack. But the highly labile subject has the potential for severe attacks, whereas the subject with little more than normal lability can only have mild asthma.

\section{Effect of age}

In this context the effect of age is important. The labile component of AR tends to decrease after adolescence and may return to normal, but not in all subjects. Some remain highly labile yet may cease to have attacks (Trevor Jones \& Jones, 1966). Presumably these subjects are always at risk, whereas those in whom lability becomes normal are not. The fixed component when dominant (Group 3), tends to persist into adult life but retrogression to Groups 2 or 1 may occur (Blackhall, 1970). If clinical symptoms persist beyond adolescence, there is a tendency, however, for progression towards Group 3 so that a relatively larger proportion of asthmatics in adult life fall into this group by comparison with Groups 1 and 2 which make up over $80 \%$ of childhood asthma.

\section{Physiology and management}

The physiological effects of therapeutic measures must be defined as far as is possible at the present time.

\section{Desensitization}

Desensitization does not appear to have any measurable effect on the labile component of AR despite the fact that the main clinical benefit is in the highly labile Group 2 subject. Nevertheless, it diminishes the tendency of the labile bronchus to constrict in response to stimuli. Thus it may be said to 'stabilize' the bronchioles (without necessarily increasing the daily values for $\mathrm{FEV}_{1}$ at rest), an effect observed with other therapeutic agents (see below). Desensitization is of relatively little value when the fixed component predominates (Group 3), possibly because in severe asthma there is sensitivity to many antigens so that desensitization to a single antigen or group of antigens makes no overall difference. The labile group on the other hand, often exhibit dominant sensitivity to a single group of antigens.

\section{Psychology}

The fact that asthma has been regarded as a psychosomatic disease follows from the labile nature of one of the components of AR. The relationship is bidirectional. The psyche may initiate nerve impulses which stimulate the bronchiolar neuromuscular mechanism, causing constriction of bronchioles within the limits set by their inherent lability. Secondly, fluctuations in ventilatory capacity may result in 'attacks' of asthma which disrupt daily routine and lead to psychological reactions, the nature of which are determined by the structure of the personality concerned.

It follows that psychotherapy cannot be expected to reduce either the labile or fixed components of AR but may 'stabilize' (in the sense defined above) the bronchioles and thus diminish the fluctuations in ventilatory capacity which cause attacks. It may seem surprising that this effect is at least as important, if not more so, in the severe asthmatic with a large fixed component as it is in the labile type. This is so because even when there is a large fixed component of AR which persistently keeps ventilatory function at a low level, the bronchioles are still abnormally labile, although this is largely obscured by the fixed component. Thus, when the overall level of ventilatory function is persistently low, small fluctuations are possible and may have disastrous effects. By minimizing these, psychotherapy may have quite a dramatic effect, even though it does notho ing to cure the basic disease.

Many factors may generate the tensions which result in constrictor effects and these tensions may be by no means evident without careful search. Important among tension-generating mechanisms is the parent-child relationship. Investigation suggests that there is a spectrum of response ranging from a parental attitude of over-protection at one end, through ambivalence to frank rejection at the other (Pinkerton, 1967; Pinkerton \& Weaver, 1970). It is noteworthy that those showing a rejective pattern are mainly Group 3 asthmatics. This may mean that the persistent, severe and long standing symptomatology in these subjects is likely to generate the more severe rejective pattern of psychological disorder.

The relationship between degree of physiological abnormality and extent of psychological disturbance may be of clinical significance. When symptomatology is florid but the physiological investigation indicates only a small increase in lability over normal (Group 1), the disproportion is an indication to probe the psychological aspect: essentially mild asthma may be used as a lever to stay off school, avoid games, etc. On the other hand if the same symptomatology is found in conjunction with a high level of lability or a large fixed component of AR (Groups 2 and 3), full weight must be given to these symptoms and management may necessitate chemotherapy. (Intal, steroids, ACTH.) 


\section{Infection and physiotherapy}

Infection may play an important part in precipitating attacks by temporarily increasing the fixed component of AR. Low grade infection is most frequent in Group 3 subjects and is difficult to eradicate, characteristically responding poorly to antibiotics. This may be due to the fact that the narrow airways with possibly impaired cilial action are incapable of evacuating secretion.

Breathing exercises per se do not influence the fixed and labile components (Buston, 1966). Control of breathing and coughing are of use primarily during periods of postural drainage used when there is an important infective factor. These sessions should be preceded by maximum dilatation with a bronchodilator drug, preferably given as an aerosol, followed by a brisk period of 2 min physical exercise (Jones, Wharton \& Buston, 1963). Bronchodilatation is necessary in order to prevent constriction caused by the act of coughing, which renders postural drainage less effective.

\section{Chemotherapy}

Bronchodilator agents are $\beta$-adrenergic stimulators or act directly on muscle, thus temporarily reducing the labile component of airways resistance. They have relatively little or no effect on the fixed component and are relatively ineffective when this predominates (Group 3). Indeed they may be dangerous if given repeatedly to these subjects on account of their cardiac action (Heaf, 1970). They are indicated primarily in Groups 1 and 2 asthma.

Salbutamol (4 mg tablet), orciprenaline sulphate $(20 \mathrm{mg})$ and isoprenaline sulphate $(10 \mathrm{mg})$ are $\beta$ adrenergic stimulants with similar action. The first is the drug of choice at the present time because of its minimal effect on the myocardium, heart rate and blood pressure. They should usually be prescribed in tablet form as abuse is less likely than when given as aerosols. It is reasonable to use an aerosol for relatively mild attacks in Groups 1 and 2 asthma, but not Group 3. These and all other bronchodilator agents should never be prescribed on a regular basis, three or four times daily, as no particular benefit appears to result and certainly no prolonged improvement in ventilatory function is achieved.

Disodium cromoglycate (Intal) has a short-lived action on the labile component and no measurable effect on the fixed comopnent (Davies, 1968; Blackhall \& Jones, 1970). It is therefore indicated in Groups 1 and 2 asthma and is usually ineffective in Group 3. However, only about $60 \%$ of highly labile asthmatics are sensitive to it and the reason for this is not known at present (Jones \& Blackhall, 1970). Although its measurable effect in reducing lability only lasts for 1-2 hr (Blackhall \& Jones,
1970), in practice, when given 6-hourly, it exerts effective action throughout the $24 \mathrm{hr}$ in sensitive subjects. Capsules should be kept in a warm place in order to avoid caking of the powder. It is essential to ensure that the patient knows how to make an effective inhalation. It is usually not possible to use it below 5-6 years of age.

In general, 'Intal Co' should be avoided. Immediate constrictor effects due to Intal are negligible in degree and frequency. The addition of isoprenaline makes it difficult to assess the response to Intal itself. Moreover, habit formation is liable to occur as with aerosols and additional capsules are then taken by the patient to treat symptoms. In fact, it is essential to explain in advance that the capsules must be taken regularly in order to prevent attacks, and are not meant to be used as treatment for an attack when it occurs. Intal capsules, therefore, should usually be prescribed in conjunction with a bronchodilator agent in the form of a tablet or syrup, the latter to be taken should an attack occur. The patient should be told that Intal will not usually help once an attack has occurred and during a severe attack it should be discontinued until it is over. Indeed, when breathless, the subject is unable to make an effective inhalation of the capsule.

$A C T H$ and steroid act primarily on the fixed component by reducing the degree of mucosal reaction and volume of sputum (Keal, 1971). An apparent increase in the labile component may be observed due to 'uncovering' of inherent lability when the fixed component is reduced. Whether they exert some stabilizing influence over the neuromuscular mechanism is uncertain but this may be so since they are effective in diminishing the frequency and severity of attacks in some highly labile Group 2 subjects. They are indicated primarily in certain Group 3 subjects. Either steroid alone or ACTH alone may prove to be the more effective agent in a given patient and only a trial of each will decide the point. In general, for long-term therapy especially in the younger individual, ACTH is preferable, whereas for intermittent therapy, steroid is preferable.

Antibiotics should be used only when there is a definite infective factor as indicated by mucopurulent sputum, or when the general signs of infection are present including fever and leucocytosis. They are not always indicated when an upper respiratory infection occurs in association with asthmatic symptoms, for the response is usually poor and the tendency is for courses to be given too frequently. If asthma is troublesome, a short course of steroid lasting 3-4 days is preferable. When infection seems important and attacks are frequent, it is reasonable to try and ration antibiotic therapy so that the worst three or four attacks occurring during a 12-month period are treated. 


\section{Management}

It is essential to explain to parents and child at the outset that whatever treatment is instituted initially, modification is usually necessary depending upon the response, and that it will take time to assess fully the various factors which may be participating. The possible role of allergens should be gone into fully and appropriate advice given, especially concerning soft furnishing in the bedroom and the role of pets and other animals. The significance of mites in house dust has recently been appreciated (Frankland, 1971). With certain exceptions (see below), the role of psychological factors need not be assessed fully at the outset. The degree to which this aspect needs investigation will vary greatly from individual to individual and the necessity for it may only become clear as therapy proceeds.

A doctor undertaking the management of an asthmatic child should always appreciate that he is embarking upon the treatment of a condition which, in most instances, will continue indefinitely and will demand patient and persistent effort in order to obtain maximum advantage from the many and varied therapeutic measures available. Little is achieved by one or two visits to even the most exalted of physicians. Continuity of therapy is absolutely essential. There is nothing worse for the morale of patient and parent than the appearance of a strange doctor, especially if his opening gambit is to demolish confidence in therapy previously given and replace it with something new. This may give the doctor short lived kudos but is disastrous in the long run. It is essential that the 'locum' or doctor taking over the care of an asthmatic should deliberately maintain existing therapy in the first instance, unless he considers it positively harmful, and refrain from criticizing it. After due appraisal of the case, he should then proceed to make whatever modifications he thinks necessary, explaining the reasons for the changes to be made.

Although at the present time there can be no unanimity of opinion about how to treat a child with asthma, there also can be no doubt that treatment must be appropriate to the particular clinical variant of the disease exhibited, and of these there are many. The following are the main ones, together with a description of appropriate management.

\section{Mild asthma}

Ventilatory function is usually normal between attacks and these are nearly always Group 1 asthmatics. It is important to ensure if the patient rates the attacks as 'mild', that there is not a large fixed component of AR making the subject a Group 3 asthmatic. Attacks respond well to bronchodilator agents using a tablet or aerosol. Intal is indicated if attacks are frequent and the response is good in most instances. Desensitization is indicated in a few. The psychological problems which arise are relatively simple. Group 3 subjects with mild attacks require different management (see below).

\section{Moderately severe and severe asthma}

The majority are labile Group 2 asthmatics and the $\mathrm{FEV}_{1}$ is normal between attacks, or becomes normal after bronchodilatation. The remainder are Group 3 asthmatics. The management of each is discussed separately below, but certain psychological points must be considered first.

Accommodation to the disease and readjustment of daily life always present problems. In some instances, consultation with a psychiatrist at the outset is desirable, but the parents may be resistant to the idea. Resistance increases if a line of treatment is commenced in which they have 'faith'. It is important, therefore, to decide this question before treatment is commenced. The decision will turn largely upon whether, on the one hand there appear to be factors inherently wrong with the environment or personal relationships, or whether on the other, symptomatology is more related to the disruption resulting from frequent or severe attacks. If the former applies, psychiatric help is indicated at the outset. If the latter appears to be the case, psychiatric involvement is preferable at a later stage, if it is then thought necessary. It is surprising however, hows often apparently florid psychological problems melt away once effective lines of treatment have been instituted and regular supervision has been established. If, despite these measures, attacks continue and there is loss of schooling, then it will be desirable to look in more detail at the psychological aspect with a view to increasing bronchiolar stability. If a psychiatrist is to be introduced at this stage, it is important to ensure that the parents have come round to the view that this is an essential and necessary step.

\section{Group 2 asthma}

Management differs in some respects according to the frequency of attacks.

\section{(1) Frequent attacks (more than one per month)}

Bronchodilator drugs should be tried in the first place. There are only two indications for their use. First, to relieve an attack and secondly, prior to exercise when this causes excessive breathlessness due to bronchoconstriction (Jones, Buston \& Wharton, 1962; Jones et al., 1963). For the latter purpose a long acting agent is preferable such as ephedrine hydrochloride, $30 \mathrm{mg}$ in the older child.

If a bronchodilator agent is being taken more than four times daily, other aspects of therapy probably need revising. There are many broncho- 
dilator agents and it is worth trying a number in order to find which seems most effective.

About $60 \%$ respond satisfactorily to Intal, four capsules daily (Jones \& Blackhall, 1970). One should te taken on waking, one at lunch-time, one on returning home from school and one on going to bed. The mid-day capsule at school tends to be omitted and although some seem to manage perfectly well with three, others require five or six daily. When attacks are primarily nocturnal, the four capsules should all be given during the period between coming home from school and retiring to bed. Experience shows that when six capsules fail to control attacks, other measures are required and there is no point in increasing the dose further, as even three or four times this dose does not seem to produce a better effect.

Steroid is indicated when attacks are frequent, troublesome and have not responded to other measures. A variety are available but prednisolone appears to be as satisfactory as the alternatives. It may be used in addition to Intal when this alone is inadequate, or in place of it when no definite benefit from Intal alone can be detected. When attacks are frequent, it is best given over a period of weeks or months rather than in the form of short courses and quite small doses are often adequate, equivalent to $2-5 \mathrm{mg}$ daily of prednisolone. More than $5 \mathrm{mg}$ should not be used except in exceptional circumstances or for short periods (2-3 weeks). When inadequate alone, ACTH should be added.

ACTH, 10-30 units two to three times per week is preferable to steroid when long-term treatment is needed, but the necessity for injections is a drawback with some children.

\section{(2) Infrequent attacks (less than one per month)}

Intal is unsuitable for this type of asthma. The response to bronchodilator agents should be explored and if this fails to give satisfactory relief, then attacks may be treated with short courses of steroid lasting 5 days to 2 weeks. The dose should be relatively high at the start and gradually tailed off according to the response. Twenty $\mathrm{mg}$ on the first day is often sufficient but higher doses are sometimes required. In some subjects with infrequent attacks, close questioning will reveal that these are occasionally extremely severe and are associated with cyanosis. This is a sinister and potentially lethal form of asthma. When this history is obtained it is advisable for the parent to have steroid available and to be instructed in exactly when and how much to give, the important point being that it be given early in the attack and in adequate dosage. Vomiting is another feature which characterizes the onset of an attack in some children and prevents the giving of bronchodilator tablets or steroid by mouth. An aminophylline suppository, intravenous aminophylline or intramuscular hydrocortisone are the alternatives.

Desensitization is always worth considering, whether attacks are frequent or infrequent. A careful history, skin tests or bronchial challenge should be used to ascertain whether there is evidence of hypersensitivity. Successful desensitization, especially to pollen, may reduce the necessity for some of the measures outlined above.

\section{Group 3 asthma}

Symptoms are persistent and there is always impaired ventilatory function. The clinical course is largely determined by the stability of the bronchial tree. Even small fluctuations in the level of ventilatory function when this is persistently low may result in frequent incapacitating attacks. On the other hand, a stable but low level of ventilatory function may be compatible with remarkable freedom from symptoms, the patient simply learning to live within his limited capacity.

The response to desensitization is poor and this is usually not worth carrying out. The presence of associated infection as indicated by mucopurulent sputum should be looked for and treated if present (see below).

Bronchodilator agents are usually contra-indicated. When used, they should be given by mouth. Aerosols tend to be habit-forming in this group in the sense that they may be used with excessive frequency. When this occurs, it will be found that ventilatory function is nearly always at an extremely low level. This is important to appreciate for two reasons. First, not only is the bronchodilator ineffective because of the large fixed component, but it may actually increase hypoxia (Palmer \& Diament, 1967; Palmer, 1969). This effect, together with $\beta$-adrenergic stimulation, may cause cardiac arrest. Secondly, if a patient is seen who has formed such a habit, his doctor does not do his duty by simply removing the aerosol, for it is not a true habit in most instances. The aerosol is taken because the subject is breathless on slight exertion due to minimal ventilatory reserve. $\mathrm{He}$ is in danger of dying if he takes his aerosol, but also if he does not. The indication is to refer for adequate investigation and treatment, which in most instances will necessitate admission to hospital.

Providing the level of ventilatory function is stable, the fact that it is low is not necessarily an indication to give steroid or ACTH. It may be preferable to accept that for most of the time the patient lives within the limits set by his ventilatory capacity. Steroid is thus reserved for exacerbations which require short courses. In this way adequate dosage can be given without the disadvantage of sideeffects from long-term therapy. 
It will be found that patients fall into two categories:

(1) Steroid-responsive. In these the response to the regimen described above for Group 2 will be found to produce appreciable improvement, which may be reflected in considerably improved ventilatory function (reduction of fixed component of AR). On the other hand, the level may remain virtuaily unchanged and the improvement be due to stabilization at a low level. In either case the response is acceptable.

(2) Steroid-resistant. These tend to be obese children or ones who put on weight readily. Even a small dose of steroid or ACTH will cause appreciable weight-gain and, in time, Cushingoid features, but only minimal improvement in ventilation and symptoms. There is no clear-cut dividing line between these and the steroid-responsive group, but at the unresponsive end of the spectrum, it is important to appreciate that no matter how much the steroid dose is pushed, normal ventilatory function is never achieved and most of the improvement comes with quite modest dosage, indicating that the doseresponse curve soon flattens out. A dose of $5 \mathrm{mg}$ of prednisolone daily plus 10-20 units ACTH three times weekly usually should not be exceeded. In the frankly resistant group, steroid and ACTH should be avoided if at all possible despite poor ventilatory function.

\section{The therapeutic failure}

The small but important group of asthmatic children (nearly all Group 3) who, despite all possible care fail to respond satisfactorily to treatment require special mention. Ventilatory function is continuously or intermittently at a low level. Home life and schooling are disrupted. Most of the mortality in asthma lies here. All concerned must appreciate the seriousness of the situation and hence accept that at least for the time being, the child will have to live differently from his fellows and special provision for schooling will be necessary.

It is well established that the residential treatment of asthma (e.g. in hospital or at a residential school) itself helps to stabilize the bronchioles (but does not necessarily raise the overall level of ventilatory function), quite apart from other therapeutic measures (Peshkin, 1959). This effect may depend not only on psychological factors but also physical ones (exercise causes bronchial muscle constriction and probably also increase of mucoid exudation), limitation of exposure to antigenic material and bacteria, but this is uncertain (Tuft, 1957). Residential treatment not only has these advantages but also allows more effective psychotherapy. It also ensures more continuous schooling. This global approach to treatment may be the only safe and effective way of help- ing a child through critical months or years when the pressures of school life may be considerable even $\frac{3}{8}$ for the child without asthma. The approach is not $\cong$ synonymous with 'parentectomy' for the extent of ${ }^{c} \cdot$ residential treatment is kept fluid-the child may spend weekends at home, or later, may attend his ownschool while still resident.

\section{Status asthmaticus}

This is usually defined as an asthmatic attack which persists for over $24 \mathrm{hr}$ and fails to respond ${ }^{\infty}$ to the usual measures, including aminophylline, $\vec{\circ}$ steroid, oxygen, sedation. Most attacks in practice $\overrightarrow{.}$ respond to these measures and the lack of response ${ }_{\sigma}^{\omega}$ is inadequate dosage. Hydrocortisone $100 \mathrm{mg}, 2-48$ hourly, IM may be required during the first 12-24 hr 3 of a severe attack. Bronchial lavage is indicated when there is serious evidence of hypoventilation as indi- $-\mathrm{v}$ cated by a raised $\mathrm{PCO}_{2}(>60 \mathrm{mmHg}$ ), cyanosis $\mathrm{f}$ $\left(\mathrm{PaO}_{2}<60 \mathrm{mmHg}\right)$ and a base deficit indicating tissue hypoxia. Intermittent positive pressure venti- $\rightarrow$ lation is occasionally required and is always indi-음 cated if respiratory failure threatens despite the $\rightarrow$ above measures.

\section{Associated pulmonary disease}

It is always essential to look for associated pน্口- $\vec{\theta}$ monary disease. An X-ray of the chest should always.be taken in status in order to exclude collapse of theo lung and a pneumothorax. The possibility of virulent infection of the bronchial tree, occasionally staphylococcal, should be considered and treated appropriately.

Although pulmonary disease may present with $\stackrel{\varrho}{\Rightarrow}$ the clinical picture of status, it may also present as a윽 separate non-asthmatic illness. Purulent bronchitis and lobar pneumonia may occur. Lobar collapse tends to occur in some subjects repeatedly when there is infection and bronchoscopy: bronchial suc- 3 tion and inflation of the lobe may be necessary. Pneumothorax is more often due to the unusual 3 respiratory effort than to an associated staphylo- $\delta$ coccal infection. A pleural drain and underwater? seal may be required.

Low grade purulent bronchitis is relatively com- $\rightarrow$ mon in Group 3. The sinuses should be X-rayed and을. the sputum examined for mucopus. The sensitivity of organisms isolated should be tested and appro- $\sigma$ priate antibiotics given. The help of an ear, nose and 0 throat surgeon will be required if there is evidence of ${ }_{\mathrm{W}}$ sinus infection. Owing to the poor response to antibiotics they may be given by inhalation (e.g. peni-o cillin 100,000 units $/ \mathrm{ml}$, or neomycin $50-100 \mathrm{mg} / \mathrm{ml}$ in $2 \mathrm{ml}$ water), in place of or as well as by the oral route, four times daily following the sessions of bronchodilatation and postural coughing. 


\section{Asthma under 5 years}

Attacks are frequently precipitated by upper respiratory infections and the low level of immunity to viral and bacterial infection is one of the main factors precipitating attacks. Many attacks respond to bronchodilator agents alone. If these occur chiefly at night, the bronchodilator should be combined with sedation with promethazine hydrochloride (Phenergan), 10-20 mg. The response to antibiotics is poor and these should be used sparingly. Desensitization is not indicated. Foci of infection in the upper respiratory tract should be treated. If these measures are inadequate, ACTH is sometimes necessary. Steroid should only be used to tide over a severe attack.

\section{Forme fruste of asthma}

An older child occasionally presents with excessive breathlessness on exertion as the only symptom. There are no abnormal physical signs, the X-ray is normal and ventilatory function is normal at rest. These children are sometimes extensively investigated on the ground that they may have some obscure pulmonary or cardiac condition. The diagnosis can only be established by measuring the labile component of $\mathrm{AR}$, using for example the technique of lability index measurement described above, when it is found to be abnormally high. Explanation and reassurance are all that is usually necessary. The only drug treatment required is the use of a long acting bronchodilator (ephedrine hydrochloride 30 $\mathrm{mg}$ ), prior to periods of more prolonged exercise which cause bronchoconstriction, such as playing games at school.

Case history. Girl, aged 13 years. Referred to an allergist by her doctor with a history of excessive breathlessness and wheezing on exercise since 2 years of age. No trouble at night or spontaneous attacks at any other time. Breathlessness interfered with school games. Physical examination and skin tests negative. Referred to a chest physician who investigated as follows: X-ray chest and tomograms: 'normal except for prominence of pulmonary artery and left heart border'. Bronchoscopy: 'Left main bronchus appeared narrow.' Bronchogram: 'Left main bronchus elongated, slightly narrow and displaced backwards; ? a vascular anomaly present'. Referred to cardiac clinic for angiogram with the suggestion of possible vascular compression.

At this point respiratory function was assessed. $\mathrm{FEV}_{1}=1.491, \mathrm{FVC}=1.911 \mathrm{n}: 1.93 \pm 0.46$ and $2 \cdot 10 \pm 0 \cdot 55) \mathrm{FEV} / \mathrm{FVC}=78 \%$. Maximum bronchodilatation with isoprenaline sulph. and exercise for 1 min, $\mathrm{FEV}_{1}=1.721$. Bronchoconstriction after 8 min exercise, $\mathrm{FEV}_{1}=1.00 \mathrm{l}$. Lability index $=37 \%$. Therefore Group 2 asthmatic. The radiographic findings were, in fact, of no significance.

\section{References}

Aмоs, H.E. (1971) Immunological aspects of asthma. Postgraduate Medical Journal, 47, 166.

BLACKHALL, M.I. (1970) Ventilatory function in subjects with childhood asthma who have become symptom free. Archives of Disease in Childhood, 45, 363.

Blackhall, M.I. \& JoNes, R.S. (1970) Effect of Intal on post-exercise bronchoconstruction in asthma. Disodium Cromoglycate in Allergic Airways Disease (Ed. by J. Pepys \& A. W. Frankland), Ch. 8, p. 63. Butterworths, London.

Buston, M.H. (1966) Factors affecting ventilatory function in the child with asthma. Archives of Disease in Childhood, 41, 509.

Davies, S.E. (1968) Effect of disodium cromoglycate on exercise induced asthma, British Medical Journal, 3, 593.

Frankland, A.W. (1971) Asthma and mites. Postgraduate Medical Journal, 47, 178.

HeAF, P.J.D. (1970) Deaths in asthma: A therapeutic misadventure? British Medical Bulletin, 26, 245.

JONES, R.S. (1966) Assessment of respiratory function in the asthmatic child. British Medical Journal, 2, 972.

JoNes, R.S. \& Blackhall, M.I. (1970) The role of disodium cromoglycate (Intal) in the treatment of childhood asthma. Archives of Disease in Childhood, 45, 49.

Jones, R.S., Buston, M.H. \& WhaRton, M.J. (1962) The effect of exercise on ventilatory function in the child with asthma. British Journal of Diseases of the Chest, 56, 78.

Jones, R.S., Wharton, M.J. \& Buston, M.H. (1963) The place of physical exercises and broncho-dilator drugs in the assessment of the child with asthma. Archives of Diseases of Childhood, 38, 539.

KEAL, E.E. (1971) Biochemistry and rheology of sputum in asthma. Postgraduate Medical Journal, 47, 171.

Palmer, K.N.V. (1969) Progress in asthma. Postgraduate Medical Journal, 45, 336.

Palmer, K.N.V. \& Diament, M.L. (1967) Effect of aerosol isoprenaline on blood gas tensions in severe bronchial asthma. Lancet, ii, 1232.

PeshKin, M.M. (1959) Intractable asthma of childhood. Rehabilitation at the institutional level with follow-up of 150 cases. International Archives of Allergy and Applied Immunology, 15, 91.

Pinkerton, P. (1967) Correlating physiologic with psychodynamic data in the study and management of childhood asthma. Session 2. The psychosomatic response. Journal of Psychosomatic Research, 2, 11.

Pinkerton, P. \& Weaver, C.M (1970) Childhood asthma. Modern Trends in Psychosomatic Medicine (Ed. by Oscar W. Hill), chap. 6, p. 81. Butterworths, London.

Scadding, J.G. (1969) Management of asthma. Brtiish Medical Journal, 3, 166.

TREVOR JoNES, R.H. \& JONES, R.S. (1966) Ventilatory capacity in young adults with a history of asthma in childhood. British Medical Journal, 2, 976.

TufT, H.S. (1957) The development and management of intractable asthma in childhood. American Journal of Diseases of Children, 93, 251. 